Open J. Math. Anal., Vol. 2(2018), No. 2, pp. 93 - 113

Website: https://pisrt.org/psr-press/journals/oma/

ISSN: 2616-8111 (Online) 2616-8103 (Print)

http://dx.doi.org/10.30538/psrp-oma2018.0021

\title{
ASYMPTOTIC STABILITY AND BLOW-UP OF SOLUTIONS FOR THE GENERALIZED BOUSSINESQ EQUATION WITH NONLINEAR BOUNDARY CONDITION
}

\author{
JIAN DANG, QINGYING HU, HONGWEI ZHANG ${ }^{1}$
}

\begin{abstract}
In this paper, we consider initial boundary value problem of the generalized Boussinesq equation with nonlinear interior source and boundary absorptive terms. We establish both the existence of the solution and a general decay of the energy functions under some restrictions on the initial data. We also prove a blow-up result for solutions with positive and negative initial energy respectively.
\end{abstract}

Mathematics Subject Classification: 35K05; 35K61; 35K70.

Key words and phrases: Generalized Boussinesq equation; Nonlinear boundary condition; Global existence; Blow-up; Decay.

\section{Introduction}

In this paper, we consider the following initial boundary value problem for the generalized Boussinesq equation with a nonlinear Neumann condition

$$
\left\{\begin{array}{l}
u_{t}-\Delta u_{t}-\Delta u+|u|^{q-2} u_{t}=f(u) \\
u=0, x \in \Gamma_{0} \\
\frac{\partial u}{\partial \nu}+g(u)=0, x \in \Gamma_{1} \\
u(x, 0)=u_{0}(x), x \in \Omega
\end{array}\right.
$$

where $u=u(t, x)(t \geq 0, x \in \Omega), \Delta$ denotes the Laplacian operator with respect to the $x$ variable, $\Omega$ is a bounded open subset of $R^{n}(n \geq 1)$ of class $C^{1}, \partial \Omega=$ $\Gamma_{0} \cup \Gamma_{1}, \operatorname{mes}\left(\Gamma_{0}\right)>0, \Gamma_{0} \cap \Gamma_{1}=\emptyset$, and $\frac{\partial}{\partial \nu}$ denotes the unit outer normal

Received 08-11-218. Accepted 30-11-2018.

1 Corresponding Author

(C) 2018 Jian Dang, Qingying Hu and Hongwei Zhang. This is an open access article distributed under the Creative Commons Attribution License, which permits unrestricted use, distribution, and reproduction in any medium, provided the original work is properly cited. 
derivative, $q>2$ is a positive constant, and the initial datum $u_{0}$ is a given function with the compatibility boundary condition $u_{0}=0$ on $\Gamma_{0}$ and $f(s)$ and $g(s)$ are continuous functions. For sake of simplicity, in this paper, we consider $f(s)=a|u|^{p-1} u, g(s)=b|u|^{k-1} u$, where $p>1, k>1$ and $a=b=1$.

Problem (11) was derived in [1]. This problem describes an electric breakdown in crystalline semiconductors with allowance for the linear dissipation of boundand free-charge sources [1, 2, 3, where the nonlinear Neumman boundary condition on the boundary of the semiconductor was introduced. According to the authors' knowledge, there are few works on the study of problem (11). Korpusov and Sveshnikov [4] and Makarov [5] proved a local theorem on the existence of solutions to the following problem

$$
\left\{\begin{array}{l}
u_{t}-\Delta u_{t}-\Delta u+\left(|u|^{q_{3}} u\right)_{t}=|u|^{q_{2}} u, \\
\frac{\partial u}{\partial \nu}+|u|^{q_{1}} u=0, x \in \partial \Omega=\Gamma, \\
u(x, 0)=u_{0}(x), x \in \Omega
\end{array}\right.
$$

by using the Galerkin method combined with the compactness method. By using the method of energy inequalities [6, 7], they also obtained sufficient conditions for the blow-up of solutions in a finite time interval and established upper and lower bounds for the blow-up time, provided the initial data satisfies

$$
\begin{aligned}
& \int_{\Omega}\left[\frac{1}{q_{2}+2}\left|u_{0}\right|^{q_{2}+2}-\frac{1}{2}\left|\nabla u_{0}\right|^{2}\right] d x-\frac{1}{q_{1}+2} \int_{\Gamma}\left|u_{0}\right|^{q_{1}+2} d x \\
& \geq c_{1}\left\{\int_{\Omega}\left[\frac{1}{2}\left|\nabla u_{0}\right|^{2}+\frac{q_{3}+1}{q_{3}+2}\left|u_{0}\right|^{q_{3}+2}\right] d x+\frac{q_{1}+1}{q_{1}+2} \int_{\Gamma}\left|u_{0}\right|^{q_{1}+2} d x\right\},
\end{aligned}
$$

where $c_{1}$ is a positive constant depending on $q_{1}, q_{2}, q_{3}$. In this paper, we consider both the existence of the solution and a general decay of the energy functions under some restrictions on the initial data. We also study blow-up condition of the solutions with positive and negative initial energy respectively.

Before we state and prove our results, let us recall some works related to the problem we address.

In the absence of the nonlinear diffusion term $|u|^{q-2} u_{t}$ and $g(u)=0$, problem (11) can be reduced to the following classical problem

$$
\left\{\begin{array}{l}
u_{t}-\Delta u_{t}-\Delta u=f(u), \\
\frac{\partial u}{\partial \nu}=0, \text { or } u=0, x \in \partial \Omega, \\
u(x, 0)=u_{0}(x), x \in \Omega,
\end{array}\right.
$$

The first equation in probem(3) can be called Sobolev type equation, SobolevGalpern type equation, pseudo-parabolic equation, or the Benjamin Bona Mahony Burgers' (BBM-Burgers) equation (for example, see [1, 3, 8, 9]. It also appears as a nonclassical diffusion equation in fluid mechanics, solid mechanics and heat conduction theory, for instance, see [10] and references therein. It's well known that problem (3) has been studied by many authors. A powerful technique to treat problem (3) is the so called "potential well method", which 
was established by Payne and Sattinger [11] and Sattinger [12], and then improved by Liu and Zhao 13 by introducing a family of potential wells. Recently, there are some interesting results about the global existence and blow-up of solutions for problem (3) with $f(u)=u^{p}$ in [14] where a family of potential wells is introduced to prove global existence, nonexistence and asymptotic behavior of solutions with low initial energy, while for high initial energy, finite time blowup of solutions is acquired by comparison principle. For other related works, we refer the readers to [1, 2, 3, 6, 7, 8, 10, 16, 15, 17, 18, 19, 20, 21, 22, 23, 24, and the references therein. The obtained results show that global existence and nonexistence depend roughly on $p$, the degree of nonlinearity in $f$, the dimension $n$, and the size of the initial data.

The equation in problem (1) with Dirichlet boundary condition (i.e. $g(u)=0$ ) has also been studied by many authors 1 , 2, 2, 3, 16, 25, 26, 27, 28. Korpusov and Sveshnikov et al 1, 2, 3, 16, 25, 26, gave the local strong solution and sufficient close-to-necessary conditions for the blow-up of solutions with negative initial energy using the energy approach developed by Levine [6]. Furthermore, they also considered two different abstract Cauchy problems for equations of Sobolev type. Zhang et al [27, 28, showed the exponential growth and blow-up of solutions with negative or positive initial energy by constructing differential inequality. We also refer to [29, 30, 31, 32, 33, 34, 35] for related results.

For the following parabolic equation with a nonlinear boundary condition or dynamic boundary condition

$$
\left\{\begin{array}{l}
u_{t}-\Delta u=f(u), \\
u=0, x \in \Gamma_{0}, \\
\frac{\partial u}{\partial \nu}=-Q\left(u_{t}\right)+g(u), x \in \Gamma_{1}, \\
u(x, 0)=u_{0}(x), x \in \Omega,
\end{array}\right.
$$

local well-posedness, global existence and blow-up results for the solutions have also been widely studied. For example, Levine and Smith 36 and Vitillaro 37. 38 studied local and global existence and nonexistence of the solutions to problem (4) by potential well theory. Also, we would like to mention the classical global existence and nonexistence results in 39, 40, 41, 42. For problem (4) with $Q=0$, as that in [43, if we interpret $u$ as a heat distribution in the body $\Omega$, and assume that $u \geq 0$ for the moment, noting that for ranges in which $-f$ is positive we have "absorption" of heat, while when $-f$ is negative we have "sources" of heat. The same holds for $-g$ : when $-g$ is positive we have a flow of heat through the boundary of $\Omega$ that extracts heat from the body, while in the opposite case, heat is flowing inside $\Omega$. Then, for problem (11) with $f(s)=|u|^{p-1} u, g(s)=|u|^{k-1} u, f$ can be called "sources" term and $g$ can be called "boundary absorptive" term. When term $|u|^{q-2} u_{t}$ does not present in problem (1), the same boundary condition arises in the literature in connection with the wave equation, i.e. when the operator $u_{t}-\Delta u$ in (11) is replaced by the wave operator $u_{t t}-\Delta u$. Some related problems concerning wave equations with nonlinear damping and source terms have been considered 
in 444, 45, 46, 47, 48, 49, 50, 51, 52, 53. In particular, Cavalcanti et al 44, deals with the problem

$$
\left\{\begin{array}{l}
u_{t t}-\Delta u=f(u) \\
\frac{\partial u}{\partial \nu}+u=-h\left(u_{t}\right)+g(u), x \in \partial \Omega, \\
u(x, 0)=u_{0}(x), u_{t}(0)=u_{1}, x \in \Omega
\end{array}\right.
$$

where under some assumptions imposed on the damping and source terms, they showed the well-posedness of the problem and effective optimal decay rates for the solutions. They also established a blow-up result in the case where the boundary source dominates the boundary damping and initial data are large enough. In general, methods employed to study hyperbolic problems cannot be employed to study parabolic problems, and conversely. Nevertheless, the arguments of 44 can be conveniently adapted to problem (1) without $|u|^{q-2} u_{t}$. However, there are several important differences in the proofs, which make the adaptation non-trivial. The first essential difference, with respect to [4], comes out here, since the boundary source term appearing in (5) is now a boundary absorptive term. When one combines boundary absorption and interior source terms with initial data of arbitrary size, the analysis becomes more difficult. Moreover, terms $-\Delta u_{t}$ and $|u|^{q-2} u_{t}$ differ from boundary damping term $Q\left(u_{t}\right)$ given in 44 .

In this paper, we will investigate the existence and nonexistence of global solutions to problem (1). More precisely, under appropriate assumptions imposed on the source and boundary absorption terms, we shall establish global existence of solutions by using the potential well method combined with a standard continuous argument. We will give sufficient conditions for the blow-up of solutions in a finite time interval under suitable initial data using differential inequality. It is different with the results in [4, 5. We also give a general decay of the energy by an integral inequality in 54 .

This paper is organized as follows. Section 2 is concerned with some notations and statement of assumptions. In Section 3, we prove global existence of solutions and the blow-up result for the solutions with positive and negative initial energy respectively. In Section 4, a general decay of the energy is proved.

\section{Preliminaries}

In this section, we present some materials needed in the proof of our results. We use the standard Lebesgue space $L^{p}(\Omega)(1<p<\infty)$ and Soblev space $H^{1}(\Omega)$ with their usual scalar products and norms. Moreover, we denote $\|u\|_{L^{p}(\Omega)}=$ $\|u\|_{p}$ and $\|u\|_{L^{p}\left(\Gamma_{1}\right)}=\|u\|_{p, \Gamma_{1}}$ for $1 \leq p \leq \infty$, and the Hilbert space $H_{\Gamma_{0}}^{1}(\Omega):=$ $\left\{u \in H^{1}(\Omega): u_{\mid \Gamma_{0}}=0\right\},\|u\|_{H_{\Gamma_{0}}^{1}}^{2}=\|\nabla u\|_{2}^{2}+\|u\|_{2}^{2}$, where $u_{\mid \Gamma_{0}}$ stands for the restriction of the trace of $u$ on $\partial \Omega$ to $\Gamma_{0}$, and in particular, we denote $\|u\|_{2}=\|u\|$ and $\|u\|_{2, \Gamma_{1}}=\|u\|_{\Gamma_{1}}$. Since meas $\left(\Gamma_{0}\right)>0$, a Poincare-type inequality holds and consequently $\|\nabla u\|$ is an equivalent norm in $H_{\Gamma_{0}}^{1}(\Omega)$. The constants $C$ used 
throughout this paper are positive generic constants, which may be various in different occurrences.

We assume that

$$
\begin{array}{r}
1<p \leq \frac{n+2}{n-2}, 1<q \leq \frac{n}{n-2} \text { if } n \geq 3 ; \\
p>1, q>1 \text { if } n=1,2 ; p>\max \{q-1, k\}>1 .
\end{array}
$$

Then, we have the Soblev embedding $H_{\Gamma_{0}}^{1}(\Omega) \hookrightarrow L^{p+1}(\Omega)$ and the trace-Soblev embedding $H_{\Gamma_{0}}^{1}(\Omega) \hookrightarrow L^{k+1}\left(\Gamma_{1}\right)$. In these cases, the embedding constants denote $c_{*}, B_{*}$ respectively, i.e.

$$
\|u\|_{p+1} \leq c_{*} \mid\|u\|_{H_{\Gamma_{0}}^{1}(\Omega)},\|u\|_{k+1, \Gamma_{1}} \leq B_{*}\|u\|_{H_{\Gamma_{0}}^{1}(\Omega)} .
$$

A function $u(x, t)$ of class $H^{1}\left(0, T ; H_{\Gamma_{0}}^{1}(\Omega)\right)$ is called a weak generalized solution of problem (1) if it satisfies the equation

$$
\begin{aligned}
& \left(u_{t}, \phi\right)+\left(\nabla u_{t}, \nabla \phi\right)+(\nabla u, \nabla \phi)+\int_{\Omega}|u|^{q-2} u_{t} \phi d x-\int_{\Omega}|u|^{p-1} u \phi d x \\
& +\int_{\Gamma_{1}}|u|^{k-1} u \phi d x+k \int_{\Gamma_{1}}|u|^{k-1} u_{t} \phi d x=0
\end{aligned}
$$

for any $\phi \in H_{\Gamma_{0}}^{1}(\Omega)$, and almost all $t \in[0, T]$ and the initial condition $u(x, 0)=$ $u_{0}(x)$ (see [4, 5]).

Theorem 2.1. Let $u_{0} \in H^{1}\left(0, T ; H_{\Gamma_{0}}^{1}(\Omega)\right)$ and $p, q, k$ satisfy (6), then problem (11) has a unique weak generalized solution on $\left[0, T_{0}\right)$ for some $T_{0}>0$, and we have either $T_{0}=+\infty$ or $T_{0}<+\infty$ and

$$
\lim _{t \rightarrow T_{0}^{-}} \sup \|u\|_{H_{\Gamma_{0}}^{1}(\Omega)}^{2}=+\infty .
$$

Theorem 2.1 can be easily established by combining the argument of [55], Theorem 1 and Theorem 2 in [4, [5], thus we omit it.

We define the functional that plays as the "potential energy"

$$
\begin{aligned}
& E(t)=E(u)=\frac{1}{2}\|\nabla u\|^{2}-\frac{1}{p+1}\|u\|_{p+1}^{p+1}+\frac{1}{k+1}\|u\|_{k+1, \Gamma_{1}}^{k+1} \\
& =\frac{1}{2}\|u\|_{H_{\Gamma_{0}}^{1}(\Omega)}^{2}-\frac{1}{p+1}\|u\|_{p+1}^{p+1}+\frac{1}{k+1}\|u\|_{k+1, \Gamma_{1}}^{k+1},
\end{aligned}
$$

and the Nehari functional

$$
I(u)=\|u\|_{H_{\Gamma_{0}}^{1}(\Omega)}^{2}-\|u\|_{p+1}^{p+1}+\|u\|_{k+1, \Gamma_{1}}^{k+1} .
$$

We also have the following identy

$$
E^{\prime}(t)=-\left.\frac{1}{2}|| u_{t}\right|_{H_{\Gamma_{0}}^{1}(\Omega)} ^{2}-\int_{\Omega}|u|^{q-2} u_{t}^{2} d x-k \int_{\Gamma_{1}}|u|^{k-1} u_{t}^{2} d x \leq 0 .
$$

In the sequel, a crucial role is played by the Nehari manifold to $I$, which is

$$
N=\left\{u \in H_{\Gamma_{0}}^{1}(\Omega) \mid I(u)=0,\|u\|_{H_{\Gamma_{0}}^{1}(\Omega)} \neq 0\right\},
$$


and we can readily give the mountain-pass level $d$ by $d=\inf _{u \in N} E(u)$.

Next, we show some properties related to functions $E(u)$ and $I(u)$ in the following lemmas.

Lemma 2.2. Let $u \in H_{\Gamma_{0}}^{1}(\Omega),\|u\|_{H_{\Gamma_{0}}^{1}(\Omega)} \neq 0$ and (6) hold, then (i) $\lim _{\lambda \rightarrow 0} E(\lambda u)=0, \lim _{\lambda \rightarrow+\infty} E(\lambda u)=-\infty$;

(ii) In the interval $0<\lambda<\infty$, there exists a unique $\lambda_{0}=\lambda_{0}(u)>0$ such that $\left.\frac{d}{d \lambda} E(\lambda u)\right|_{\lambda=\lambda_{0}}=0$;

(iii) $E(\lambda u)$ is increasing on $0<\lambda \leq \lambda_{0}$, decreasing on $\lambda_{0} \leq \lambda<+\infty$ and takes the maximum at $\lambda=\lambda_{0}$;

(iv) $I(\lambda u)>0$, for $0<\lambda<\lambda_{0} ; I(\lambda u)<0$, for $\lambda>\lambda_{0}$ and $I\left(\lambda_{0} u\right)=0$.

Proof. (i) The conclusion follows from

$$
E(\lambda u)=\frac{\lambda^{2}}{2}\|u\|_{H_{\Gamma_{0}}^{1}(\Omega)}^{2}-\frac{\lambda^{p+1}}{p+1}\|u\|_{p+1}^{p+1}+\frac{\lambda^{k+1}}{k+1}\|u\|_{k+1, \Gamma_{1}}^{k+1} .
$$

(ii) First, note that

$$
\frac{d}{d \lambda} E(\lambda u)=\lambda\|u\|_{H_{\Gamma_{0}}^{1}(\Omega)}^{2}-\lambda^{p}\|u\|_{p+1}^{p+1}+\lambda^{k}\|u\|_{k+1, \Gamma_{1}}^{k+1}=0, \lambda>0
$$

is equivalent to

$$
\lambda^{p-1}|| u\left\|_{p+1}^{p+1}-\lambda^{k-1}|| u\right\|_{k+1, \Gamma_{1}}^{k+1}=\|u\|_{H_{\Gamma_{0}}^{1}(\Omega)}^{2} .
$$

Let

$$
\begin{aligned}
h(\lambda) & =\lambda^{p-1}\|u\|_{p+1}^{p+1}-\lambda^{k-1}\|u\|_{k+1, \Gamma_{1}}^{k+1} \\
& =\lambda^{k-1}\left(\lambda^{p-k}\|u\|_{p+1}^{p+1}-\|u\|_{k+1, \Gamma_{1}}^{k+1}\right) \\
& =\lambda^{k-1} h_{1}(\lambda),
\end{aligned}
$$

where $h_{1}(\lambda)=\lambda^{p-k}\|u\|_{p+1}^{p+1}-\|u\|_{k+1, \Gamma_{1}}^{k+1}$. Note that $h_{1}(\lambda)$ is increasing on $0<$ $\lambda<\infty, \lim _{\lambda \rightarrow 0^{+}} h_{1}(\lambda) \leq 0$, and $\lim _{\lambda \rightarrow+\infty} h_{1}(\lambda)=+\infty$, and hence there exists a unique $\lambda^{*}>0$ such that $h_{1}\left(\lambda^{*}\right)=0$, thereby $h\left(\lambda^{*}\right)=0, h(\lambda)<0$ for $0<\lambda<\lambda^{*}$, $h(\lambda)>0$ for $\lambda^{*}<\lambda<\infty$. Hence, for any $\|u\|_{H_{\Gamma_{0}}^{1}(\Omega)}>0$, there exists a unique $\lambda_{0}>\lambda^{*}$ such that (10) holds, and then (ii) holds.

(iii) Note that $\frac{d}{d \lambda} E(\lambda u)=\lambda\left(\|u\|_{H_{\Gamma_{0}}^{1}(\Omega)}^{2}-h(\lambda)\right)$. From the proof of (ii), it follows that if $0<\lambda<\lambda^{*}$, then $h(\lambda)<0$; if $\lambda^{*}<\lambda<\lambda_{0}$, then $0<$ $h(\lambda)<\|u\|_{H_{\Gamma_{0}}^{1}(\Omega)}^{2}$; and if $\lambda_{0}<\lambda<\infty$, then $h(\lambda)>\|u\|_{H_{\Gamma_{0}}^{1}(\Omega)}^{2}$. From this, the conclusion of (iii) holds.

(iv)The conclusion follows from the proof of (iii) and

$$
I(\lambda u)=\lambda^{2}\|u\|_{H_{\Gamma_{0}}^{1}(\Omega)}^{2}-\lambda^{p+1}\|u\|_{p+1}^{p+1}+\lambda^{k+1}\|u\|_{k+1, \Gamma_{1}}^{k+1}=\lambda \frac{d}{d \lambda} E(\lambda u) .
$$

This completes the proof of Lemma 2.2. 
Now, we define

$$
F(x)=\frac{1}{2} x^{2}-\frac{c_{*}^{p+1}}{p+1} x^{p+1}-\frac{B_{*}^{k+1}}{k+1} x^{k+1},
$$

and let $r_{0}$ be the unique real root of equation $F^{\prime}(x)=0$. We easily verify that $r_{0}$ is the unique real root of equation $\phi(x)=1$, where $\phi(x)=c_{*}^{p+1} x^{p-1}+B_{*}^{k+1} x^{k-1}$, then $\phi\left(r_{0}\right)=c_{*}^{p+1} r_{0}^{p-1}+B_{*}^{k+1} r_{0}^{k-1}=1$. It can be checked that $r_{0}$ is a point of local maximum for $F(x)$ (see 44 for more details). Accordingly, let us define $E_{1}$ as

$$
E_{1}=F\left(r_{0}\right)=\frac{1}{2} r_{0}^{2}-\frac{c_{*}^{p+1}}{p+1} r_{0}^{p+1}-\frac{B_{*}^{k+1}}{k+1} r_{0}^{k+1} .
$$

Lemma 2.3. Let (6) hold, then (i) if $0<\|u\|_{H_{\Gamma_{0}}^{1}(\Omega)}<r_{0}$, then $I(u)>0$; (ii) if $I(u)<0$, then $\|u\|_{H_{\Gamma_{0}}^{1}(\Omega)}>r_{0}$; (iii) if $I(u)=0$ and $\|u\|_{H_{\Gamma_{0}}^{1}(\Omega)} \neq 0$, i.e. $u \in N$, then $\|u\|_{H_{\Gamma_{0}}^{1}(\Omega)} \geq r_{0}$.

Proof. (i) Since $\phi(x)$ is a strictly increasing function in $\left(0, r_{0}\right)$, from

$$
0<\|u\|_{H_{\Gamma_{0}}^{1}(\Omega)}<r_{0},
$$

we get $\phi\left(\|u\|_{H_{\Gamma_{0}}^{1}(\Omega)}\right)<\phi\left(r_{0}\right)$ and

$$
\begin{aligned}
& I(u)=\|u\|_{H_{\Gamma_{0}}^{1}(\Omega)}^{2}-\|u\|_{p+1}^{p+1}+\|u\|_{k+1, \Gamma_{1}}^{k+1} \\
& \geq\|u\|_{H_{\Gamma_{0}}^{1}(\Omega)}^{2}-\|u\|_{p+1}^{p+1}-\|u\|_{k+1, \Gamma_{1}}^{k+1} \\
& =\|u\|_{H_{\Gamma_{0}}^{1}(\Omega)}^{2}\left(1-c_{*}^{p+1}\|u\|_{H_{\Gamma_{0}}^{1}(\Omega)}^{p-1}-B_{*}^{k+1}\|u\|_{H_{\Gamma_{0}}^{1}(\Omega)}^{k-1}\right) \\
& =\|u\|_{H_{\Gamma_{0}}^{1}(\Omega)}^{2}\left(\phi\left(r_{0}\right)-\phi\left(\|u\|_{H_{\Gamma_{0}}^{1}(\Omega)}\right)\right)>0 .
\end{aligned}
$$

(ii) Condition $I(u)<0$ gives

$$
\begin{aligned}
& \phi\left(r_{0}\right)\|u\|_{H_{\Gamma_{0}}^{1}(\Omega)}^{2}=\|u\|_{H_{\Gamma_{0}}^{1}(\Omega)}^{2} \\
& <\|u\|_{p+1}^{p+1}-\|u\|_{k+1, \Gamma_{1}}^{k+1}<\|u\|_{p+1}^{p+1}+\|u\|_{k+1, \Gamma_{1}}^{k+1} \\
& \leq\left(c_{*}^{p+1}\|u\|_{H_{\Gamma_{0}}^{1}(\Omega)}^{p-1}+B_{*}^{k+1}\|u\|_{H_{\Gamma_{0}}^{1}(\Omega)}^{k-1}\right)\|u\|_{H_{\Gamma_{0}}^{1}(\Omega)}^{2}=\phi\left(\|u\|_{H_{\Gamma_{0}}^{1}(\Omega)}\right)\|u\|_{H_{\Gamma_{0}}^{1}(\Omega)}^{2},
\end{aligned}
$$

which implies $\|u\|_{H_{\Gamma_{0}}^{1}(\Omega)} \neq 0$ and $\|u\|_{H_{\Gamma_{0}}^{1}(\Omega)}>r_{0}$ by the monotonicity of $\phi$. (iii) If $I(u)=0$ and $\|u\|_{H_{\Gamma_{0}}^{1}(\Omega)} \neq 0$, then

$$
\begin{aligned}
& \phi\left(r_{0}\right)\|u\|_{H_{\Gamma_{0}}^{1}(\Omega)}^{2}=\|u\|_{H_{\Gamma_{0}}^{1}(\Omega)}^{2}=\|u\|_{p+1}^{p+1}-\|u\|_{k+1, \Gamma_{1}}^{k+1} \\
& \leq\|u\|_{p+1}^{p+1}+\|u\|_{k+1, \Gamma_{1}}^{k+1} \leq \phi\left(\|u\|_{H_{\Gamma_{0}}^{1}(\Omega)}\right)\|u\|_{H_{\Gamma_{0}}^{1}(\Omega)}^{2},
\end{aligned}
$$

and from the monotonicity of $\phi$, we get $\|u\|_{H_{\Gamma_{0}}^{1}(\Omega)}>r_{0}$.

Lemma 2.4. $d \geq d_{0}=\left(\frac{1}{2}-\frac{1}{p+1}\right) r_{0}^{2}=\frac{p-1}{2(p+1)} r_{0}^{2}$. 
Proof. For $u \in N$ (or $I(u)=0$ and $\|u\|_{H_{\Gamma_{0}}^{1}(\Omega)} \neq 0$ ), by Lemma 2.3, we have $\|u\|_{H_{\Gamma_{0}}^{1}(\Omega)}>r_{0}$. Hence

$$
\begin{aligned}
& E(u) \geq \frac{1}{2}\|u\|_{H_{\Gamma_{0}}^{1}(\Omega)}^{2}+\frac{1}{p+1}\left(-\|u\|_{p+1}^{p+1}+\|u\|_{k+1, \Gamma_{1}}^{k+1}\right) \\
& =\left(\frac{1}{2}-\frac{1}{p+1}\right)\|u\|_{H_{\Gamma_{0}}^{1}(\Omega)}^{2}+\frac{1}{p+1} I(u) \\
& =\left(\frac{1}{2}-\frac{1}{p+1}\right)\|u\|_{H_{\Gamma_{0}}^{1}(\Omega)}^{2} \geq\left(\frac{1}{2}-\frac{1}{p+1}\right) \lambda_{0}^{2},
\end{aligned}
$$

which gives $d \geq d_{0}$.

Remark 2.5. Noting the definition of $d$ and the fact that

$$
\begin{aligned}
& E(u)=\frac{1}{2}\|u\|_{H_{\Gamma_{0}}^{1}(\Omega)}^{2}-\frac{1}{p+1}\|u\|_{p+1}^{p+1}+\frac{1}{k+1}\|u\|_{k+1, \Gamma_{1}}^{k+1} \\
& \geq \frac{1}{2}\|u\|_{H_{\Gamma_{0}}^{1}(\Omega)}^{2}-\frac{c_{*}^{p+1}}{p+1}\|u\|_{H_{\Gamma_{0}}^{1}(\Omega)}^{p+1}-\frac{B_{*}^{k+1}}{k+1}\|u\|_{H_{\Gamma_{0}}^{1}(\Omega)}^{k+1}=F\left(\|u\|_{H_{\Gamma_{0}}^{1}(\Omega)}\right),
\end{aligned}
$$

we know $d \geq E_{1}$.

Now we define the subsets of $H_{\Gamma_{0}}^{1}(\Omega)$ related to problem (1)-(3). Set

$W=\left\{u \in H_{\Gamma_{0}}^{1}(\Omega) \mid E(u)<d, I(u)>0\right\}, V=\left\{u \in H_{\Gamma_{0}}^{1}(\Omega) \mid E(u)<d, I(u)<0\right\}$.

Lemma 2.6. If $u_{0} \in H_{\Gamma_{0}}^{1}(\Omega), 0<E(0)<d$, and $u$ is a weak solution of problem (11) -(3), then (i) $u \in W$ if $I\left(u_{0}\right)>0$ or $\|u\|_{H_{\Gamma_{0}}^{1}(\Omega)}=0$; (ii) $u \in V$ if $I\left(u_{0}\right)<0$.

Proof. We only prove (i), and the proof for (ii) is similar. We are going to prove that $u \in W$ for $0<t<T_{0}$. From (9), we have

$$
\begin{aligned}
& E(u(t))+\int_{0}^{t}\left[\left\|u_{t}\right\|_{H_{\Gamma_{0}}^{1}(\Omega)}^{2}+\int_{\Omega}|u|^{q-2} u_{t}^{2} d x+k \int_{\Gamma_{1}}|u|^{k-1} u_{t}^{2} d x\right] d s \\
& =E(0)<d, \text { for any } t \in\left[0, T_{0}\right),
\end{aligned}
$$

which implies $E(u(t))<d$. To prove that $u \in W$ for $0<t<T_{0}$, we argue by contradiction. Indeed, if it is not the case, there would exist $t_{0} \in\left(0, T_{0}\right)$ such that $u\left(t_{0}\right) \in N$, and by the definition of $d=\inf _{u \in N} E(u)$, one has $d<E\left(t_{0}\right) \leq d$, then we reach to a contradiction.

\section{Global existence and blow-up of solutions}

In this section, we prove the global existence and blow-up of solutions to problem (11).

Theorem 3.1. Let $u_{0} \in H_{\Gamma_{0}}^{1}(\Omega), 0<E(0)<d, I\left(u_{0}\right)>0$ or $\|u\|_{H_{\Gamma_{0}}^{1}(\Omega)}=0$, and $p, q, k$ satisfies (6), then the weak solution u to problem (11) in Theorem 2.1 can be extended to $(0, \infty)$. 
Proof. By Lemma 2.5, we have $u \in W$, then $I(u)>0$ and $E(u)<d$ for all $t \in\left(0, T_{0}\right)$. Therefore,

$$
\begin{aligned}
& d>E(u)=\frac{1}{2}\|u\|_{H_{\Gamma_{0}}^{1}(\Omega)}^{2}-\frac{1}{p+1}\|u\|_{p+1}^{p+1}+\frac{1}{k+1}\|u\|_{k+1, \Gamma_{1}}^{k+1} \\
& >\left(\frac{1}{2}-\frac{1}{p+1}\right)\|u\|_{H_{\Gamma_{0}}^{1}(\Omega)}^{2}+\frac{1}{p+1} I(u) \\
& >\left(\frac{1}{2}-\frac{1}{p+1}\right)\|u\|_{H_{\Gamma_{0}}^{1}(\Omega)}^{2}
\end{aligned}
$$

for all $t \in\left(0, T_{0}\right)$. Then, (13) and (7) imply

$$
\begin{aligned}
\|u\|_{H_{\Gamma_{0}}^{1}(\Omega)}^{2} & <\frac{2(p+1) d}{p-1},\|u\|_{p+1}^{p+1} \\
& <c_{*}^{p+1}\left(\frac{2(p+1) d}{p-1}\right)^{\frac{p+1}{2}},\|u\|_{k+1, \Gamma_{1}}^{k+1} \\
& <B_{*}^{k+1}\left(\frac{2(p+1) d}{p-1}\right)^{\frac{k+1}{2}}
\end{aligned}
$$

for all $t \in(0, T)$. By (8) and the definition of $E(u)$, we have

$$
\frac{1}{2}\left\|u_{t}\right\|^{2}+\frac{1}{2}\|u\|_{H_{\Gamma_{0}}^{1}(\Omega)}^{2} \leq E(0)+\frac{1}{p+1}\|u\|_{p+1}^{p+1}-\frac{1}{k+1}\|u\|_{k+1, \Gamma_{1}}^{k+1}<C<+\infty(15
$$

for all $t \in(0, T)$. It follows from (15) and from a standard continuous argument that local weak solution $u$ furnished by Theorem 2.1 can be extended to the whole internal $[0, \infty)$, that is to say, $u$ is a global solution.

Theorem 3.2. Suppose that assumption (6) holds, $u(0)=u_{0} \in H_{\Gamma_{0}}^{1}(\Omega)$ and $u$ is a local solution of probelem (11). If $E(0)<0$, then the solution of the system (11) blows up in finite time.

Proof. We set

$$
H(t)=-E(t) .
$$

By the definition of $H(t)$ and (9),

$$
H^{\prime}(t)=-E^{\prime}(t) \geq 0 .
$$

Consequently, by $E(0)<0$, we have

$$
H(0)=-E(0)>0 .
$$

It is clear that by (17) and (18)

$$
0<H(0) \leq H(t) .
$$

By (16) and the expression of $E(t)$,

$$
H(t)-\frac{1}{p+1}\|u\|_{p+1}^{p+1}+\frac{1}{k+1}\|u\|_{k+1, \Gamma_{1}}^{k+1}=-\frac{1}{2}\|\nabla u\|^{2}<0 .
$$


One implies

$$
\begin{aligned}
& 0<H(0) \leq H(t) \leq \frac{1}{p+1}\|u\|_{p+1}^{p+1}-\frac{1}{k+1}\|u\|_{k+1, \Gamma_{1}}^{k+1} \\
& \leq \frac{1}{p+1}\|u\|_{p+1}^{p+1} \leq \frac{1}{p+1}\|u\|_{p+1}^{p+1}+\frac{1}{k+1}\|u\|_{k+1, \Gamma_{1}}^{k+1} .
\end{aligned}
$$

Let us define the functional

$$
L(t)=H^{1-\sigma}(t)+\frac{\epsilon}{2}\|\nabla u\|^{2}+\frac{\epsilon}{2}\|u\|^{2},
$$

where $\epsilon>0$ will be fixed in later and $0<\sigma \leq \frac{p+1-q}{p+1}$ (this can be done since $q-1<p$ ). By taking the time derivative of (21), using problem (11), and performing several integration by parts, we get

$$
\begin{aligned}
& L^{\prime}(t)=(1-\sigma) H^{-\sigma}(t) H^{\prime}(t)+\epsilon \int_{\Omega} \nabla u \nabla u_{t} d x+\epsilon \int_{\Omega} u u_{t} d x \\
& =(1-\sigma) H^{-\sigma}(t) H^{\prime}(t)+\epsilon \int_{\Omega}\left[u u_{t}-u \Delta u_{t}\right] d x+\epsilon \int_{\Gamma_{1}} u_{t} \frac{\partial u}{\partial \nu} d x \\
& =(1-\sigma) H^{-\sigma}(t) H^{\prime}(t)+2 \epsilon H(t)+2 \epsilon E(t)-\epsilon\|\nabla u\|^{2} \\
& +\epsilon\|u\|_{p+1}^{p+1}+\epsilon\|u\|_{k+1, \Gamma_{1}}^{k+1}-\epsilon \int_{\Omega}|u|^{q-2} u u_{t} d x+\epsilon \int_{\Gamma_{1}} u_{t}|u|^{k-1} u d x \\
& =(1-\sigma) H^{-\sigma}(t) H^{\prime}(t)+2 \epsilon H(t)+\epsilon\left(1-\frac{2}{p+1}\right)\|u\|_{p+1}^{p+1} \\
& +\epsilon\left(1+\frac{2}{k+1}\right)\|u\|_{k+1, \Gamma_{1}}^{k+1}-\epsilon \int_{\Omega}|u|^{q-2} u u_{t} d x+\epsilon \int_{\Gamma_{1}} u_{t}|u|^{k-1} u d x .
\end{aligned}
$$

To estimate the last two terms in the right-hand side of (23), by the following Young's inequality

$$
a b \leq \delta^{-1} a^{2}+\delta b^{2},
$$

we deduce that, for any $\delta_{1}>0$ and $\delta_{2}>0$,

$$
\begin{aligned}
& \int_{\Omega}|u|^{q-2} u u_{t} d x=\int_{\Omega}\left(|u|^{\frac{q-2}{2}} u_{t}\right)\left(|u|^{\frac{q-2}{2}} u\right) d x \leq \delta_{1}^{-1} \int_{\Omega}|u|^{q-2} u_{t}^{2} d x+\delta_{1} \int_{\Omega}|u|^{q} d x, \\
& \int_{\Gamma_{1}}|u|^{k-1} u u_{t} d x \\
& =\int_{\Gamma_{1}}\left(|u|^{\frac{k-1}{2}} u_{t}\right)\left(|u|^{\frac{k-1}{2}} u\right) d x \leq \delta_{2}^{-1} \int_{\Gamma_{1}}|u|^{k-1} u_{t}^{2} d x+\delta_{2} \int_{\Gamma_{1}}|u|^{k+1} d x .
\end{aligned}
$$

Therefore, we have

$$
\begin{aligned}
& L^{\prime}(t) \geq(1-\sigma) H^{-\sigma}(t) H^{\prime}(t)+2 \epsilon H(t)+\epsilon\left(1-\frac{2}{p+1}\right)\|u\|_{p+1}^{p+1} \\
& +\epsilon\left(1+\frac{2}{k+1}\right)\|u\|_{k+1, \Gamma_{1}}^{k+1}
\end{aligned}
$$




$$
-\epsilon \delta_{1}\|u\|_{q}^{q}-\epsilon \delta_{2}\|u\|_{k+1, \Gamma_{1}}^{k+1}-\epsilon \delta_{1}^{-1} \int_{\Omega}|u|^{q-2} u_{t}^{2} d x-\epsilon \delta_{2}^{-1} \int_{\Gamma_{1}}|u|^{k-1} u_{t}^{2} d x .
$$

By choosing $\delta_{1}$ such that $\delta_{1}^{-1}=M_{1} H^{-\sigma}(t)$ for $M_{1}$ enough large constants to be fixed later, and noting that

$$
-\int_{\Omega}|u|^{q-2} u_{t}^{2} d x \geq-H^{\prime}(t),-\int_{\Gamma_{1}}|u|^{k-1} u_{t}^{2} d x \geq-H^{\prime}(t)
$$

by (9) and (17), we have

$$
\begin{aligned}
& L^{\prime}(t) \geq\left[\left(1-\sigma-\epsilon M_{1}\right) H^{-\sigma}(t)-\epsilon \delta_{2}^{-1}\right] H^{\prime}(t)+2 \epsilon H(t)+\epsilon\left(1-\frac{2}{p+1}\right)\|u\|_{p+1}^{p+1} \\
& +\epsilon\left(1+\frac{2}{k+1}-\delta_{2}\right)\|u\|_{k+1, \Gamma_{1}}^{k+1}-\epsilon M_{1}^{-1} H^{\sigma}(t)\|u\|_{q}^{q} .
\end{aligned}
$$

Taking into account (21) and the embedding $L^{p+1}(\Omega) \hookrightarrow L^{q}(\Omega)$, we get

$$
H^{\sigma}(t)\|u\|_{q}^{q} \leq C_{1}\|u\|_{p+1}^{(p+1) \sigma}\|u\|_{q}^{q} \leq C_{2}\|u\|_{p+1}^{(p+1) \sigma+q},
$$

for some positive constants $C_{1}$ and $C_{2}$. Now apply the inequality

$$
x^{l} \leq(x+1) \leq\left(1+\frac{1}{z}\right)(x+z), x \geq 0,0 \leq l \leq 1, z>0,
$$

in particular, taking $x=\|u\|_{p+1}^{p+1}, l=\frac{(p+1) \sigma+q}{p+1}, z=H(0)$, we obtain

$$
\|u\|_{p+1}^{(p+1) \sigma+q}=\left(\|u\|_{p+1}^{p+1}\right)^{l} \leq\left(1+\frac{1}{H(0)}\right)\left(\|u\|_{p+1}^{p+1}+H(0)\right) \leq C_{3}\|u\|_{p+1}^{p+1} .
$$

where we have used the fact that $0<\frac{q}{p+1}<1,0<\sigma \leq \frac{p+1-q}{p+1}$ and (21).

By (25), (26) and (28), we have

$$
\begin{aligned}
& L^{\prime}(t) \geq\left[\left(1-\sigma-\epsilon M_{1}\right) H^{-\sigma}(t)-\epsilon \delta_{2}^{-1}\right] H^{\prime}(t)+2 \epsilon H(t) \\
& +\epsilon\left(1-\frac{2}{p+1}-C_{3} M_{1}^{-1}\right)\|u\|_{p+1}^{p+1}+\epsilon\left(1+\frac{2}{k+1}-\delta_{2}\right)\|u\|_{k+1, \Gamma_{1}}^{k+1} .
\end{aligned}
$$

Now, we take $\delta_{2}$ such that $1+\frac{2}{k+1}-\delta_{2}>0$, and we take $M_{1}$ large enough such that $1-\frac{2}{p+1}-C_{3} M_{1}^{-1}=C_{4}>0$. Once $M_{1}$ and $\delta_{2}$ are fixed, we can pick $\epsilon$ small enough such that

$$
\begin{aligned}
& 1-\sigma-\epsilon M_{1}>0, \\
& \left(1-\sigma-\epsilon M_{1}\right) H^{-\sigma}(t)-\epsilon \delta_{2}^{-1}>\left(1-\sigma-\epsilon M_{1}\right) H^{-\sigma}(0)-\epsilon \delta_{2}^{-1}>0,
\end{aligned}
$$

where we have used the fact that $H^{-\sigma}(t)>H^{-\sigma}(0)$. Then there exist $C_{5}>0$ such that (29) becomes

$$
L^{\prime}(t) \geq C_{5}\left(H(t)+\|u\|_{p+1}^{p+1}+\|u\|_{k+1, \Gamma_{1}}^{k+1}\right) .
$$

Then, we have

$$
L(t) \geq L(0) \geq 0 .
$$


On the other hand, by the definition of $L(t)$ and (20), we have

$$
\begin{aligned}
& L(t)=H^{1-\sigma}(t)-\epsilon\left(H(t)-\frac{1}{p+1}\|u\|_{p+1}^{p+1}+\frac{1}{k+1}\|u\|_{k+1, \Gamma_{1}}^{k+1}\right)+\frac{\epsilon}{2}\|u\|^{2} \\
& \leq(1-\epsilon) H^{1-\sigma}(t)+\frac{\epsilon}{p+1}\|u\|_{p+1}^{p+1}-\frac{\epsilon}{k+1}\|u\|_{k+1, \Gamma_{1}}^{k+1}+\frac{\epsilon}{2}\|u\|^{2} \\
& \leq(1-\epsilon) H^{1-\sigma}(t)+\frac{\epsilon}{p+1}\|u\|_{p+1}^{p+1}+\frac{\epsilon}{2}\|u\|^{2},
\end{aligned}
$$

where we have used the fact $H(t) \geq H^{1-\sigma}(t)$ (this can be ensured by (18), (19), $0<\sigma<1$ and that $E(0)$ is sufficient negative).

By the inequality (27) with $x=\|u\|_{p+1}^{\frac{p+1}{1+\sigma}}, l=1-\sigma<1, z=H^{\frac{1}{1-\sigma}}(0)$, we have

$$
\|u\|_{p+1}^{p+1}=\left(\|u\|_{p+1}^{\frac{p+1}{1-\sigma}}\right)^{1-\sigma} \leq\left(1+\frac{1}{H^{\frac{1}{1-\sigma}}(0)}\right)\left(\|u\|_{p+1}^{\frac{p+1}{1-\sigma}}+H^{\frac{1}{1-\sigma}}(0)\right) \leq C_{6}\|u\|_{p+1}^{\frac{p+1}{1-\sigma}} .
$$

Therefore, we get

$$
L(t) \leq(1-\epsilon) H^{1-\sigma}(t)+C_{6}\|u\|_{p+1}^{\frac{p+1}{1-\sigma}}+\frac{\epsilon}{2}\|u\|^{2} .
$$

Then, by the embedding $L^{p+1}(\Omega) \hookrightarrow L^{2}(\Omega)$, we have, for fixed $\epsilon$ sufficient small,

$$
L^{\frac{1}{1-\sigma}}(t) \leq C_{7}\left[H(t)+\|u\|_{p+1}^{p+1}+\|u\|_{p+1}^{\frac{2}{1-\sigma}}\right] .
$$

Using again the inequality (27) with $x=\|u\|_{p+1}^{p+1}, l=\frac{2}{(p+1)(1-\sigma)}<1$ (since $\left.\sigma<\frac{p+1-q}{p+1}<\frac{p-1}{p+1}\right), z=H(0)$, we have

$$
\|u\|_{p+1}^{\frac{2}{1-\sigma}}=\left(\|u\|_{p+1}^{p+1}\right)^{\frac{2}{(p+1)(1-\sigma)}} \leq\left(1+\frac{1}{H(0)}\right)\left(\|u\|_{p+1}^{p+1}+H(0)\right) \leq C_{8}\|u\|_{p+1}^{p+1} .
$$

From (32) and (33), we obtain

$$
L^{\frac{1}{1-\sigma}}(t) \leq C_{9}\left(H(t)+\|u\|_{p+1}^{p+1}\right) \leq C_{9}\left(H(t)+\|u\|_{p+1}^{p+1}+\|u\|_{k+1, \Gamma_{1}}^{k+1}\right) .
$$

Combining with (30) and (34), we arrive that

$$
L^{\prime}(t) \geq C_{10} L^{\frac{1}{1-\sigma}}(t) .
$$

Integration of (35) between 0 and $t$ gives the desired results. The theorem is proved.

In the following, we will prove that the solution will blow up provided that the initial energy $E(0)>0$. The next lemma will play an essential role in our proving and it is similar to a lemma used firstly by Vitillaro [56. Now the main idea of the proof is from Lemma 9.1 in [44]. 
Lemma 3.3. Let $u$ be a solution of problem (11). Suppose that the assumption of $k, p$ hold. Further assume that $E(0)<E_{1}$ and $\|u(0)\|_{H_{\Gamma_{0}}^{1}(\Omega)}>r_{0}$. Then there exists a constant $r_{1}>r_{0}$ such that $\|u(t)\|_{H_{\Gamma_{0}}^{1}(\Omega)} \geq r_{1}$, and

$$
\frac{1}{p+1}\|u\|_{p+1}^{p+1}+\frac{1}{k+1}\|u\|_{k+1, \Gamma_{1}}^{k+1} \geq \frac{1}{2} r_{1}^{2}-F\left(r_{1}\right)=\frac{c_{*}^{p+1}}{p+1} r_{1}^{p+1}+\frac{B_{*}^{k+1}}{k+1} r_{1}^{k+1} .
$$

Proof. We observe from (11) that

$$
E(u(t)) \geq F\left(\|u\|_{H_{\Gamma_{0}}^{1}(\Omega)}\right) .
$$

We have that $F(r)$ is increasing for $0<r<r_{0}$, decreasing for $r>r_{0}, F\left(r_{0}\right)=E_{1}$, and $\lim _{r \rightarrow+\infty} F(r)=-\infty$. Then, since $d \geq E_{1}>E(u(0)) \geq F\left(\|u(0)\|_{H_{\Gamma_{0}}^{1}(\Omega)}\right) \geq$ $F(0)=0$, there exist $r_{1}^{\prime}<r_{0}<r_{1}$, which verify

$$
F\left(r_{1}\right)=F\left(r_{1}^{\prime}\right)=E(u(0)) \text {. }
$$

Considering that $E(t)$ is non-increasing, we have

$$
E(u(t)) \leq E(u(0)) .
$$

From (37) and (38) we have

$$
F\left(\|u(0)\|_{H_{\Gamma_{0}}^{1}(\Omega)}\right) \leq E(u(0))=F\left(r_{1}\right) .
$$

Since $\|u(0)\|_{H_{\Gamma_{0}}^{1}(\Omega)}, r_{1} \in\left(r_{0},+\infty\right)$ and $F(r)$ is deceasing in this interval, from (39) one has

$$
\|u(0)\|_{H_{\Gamma_{0}}^{1}(\Omega)} \geq r_{1}
$$

In the sequel, we will prove that

$$
\|u(t)\|_{H_{\Gamma_{0}}^{1}(\Omega)} \geq r_{1} .
$$

In fact, we will argue by contradiction. Supposing that (41) does not hold, then, there exists $t^{*} \in\left(0, T_{0}\right)$ such that

$$
\left\|u\left(t^{*}\right)\right\|_{H_{\Gamma_{0}}^{1}(\Omega)}<r_{1} .
$$

If $\left\|u\left(t^{*}\right)\right\|_{H_{\Gamma_{0}}^{1}(\Omega)}>r_{0}$, then,from (36), (37) and (42), we have

$$
E\left(u\left(t^{*}\right)\right) \geq F\left(\left\|u\left(t^{*}\right)\right\|_{H_{\Gamma_{0}}^{1}(\Omega)}\right)>F\left(r_{1}\right)=E(u(0)),
$$

which contradicts (38) and proves (41). Now, if $\left\|u\left(t^{*}\right)\right\|_{H_{\Gamma_{0}}^{1}(\Omega)} \leq r_{0}$, we have, taking (40) into account, that there exists $r_{2}$ which verifies

$$
\left.\left\|u\left(t^{*}\right)\right\|_{H_{\Gamma_{0}}^{1}(\Omega)}\right) \leq r_{0}<r_{2}<r_{1} \leq\|u(0)\|_{H_{\Gamma_{0}}^{1}(\Omega)} .
$$

Consequently, from the continuity of $\|u(.)\|_{H_{\Gamma_{0}}^{1}(\Omega)}$, there exists $t^{\prime} \in\left(0, t^{*}\right)$ verifying

$$
\left\|u\left(t^{\prime}\right)\right\|_{H_{\Gamma_{0}}^{1}(\Omega)}=r_{2}
$$


From the last identity and from (36), (37) and (43), we obtain

$$
E\left(u\left(t^{\prime}\right)\right) \geq F\left(\left\|u\left(t^{\prime}\right)\right\|_{H_{\Gamma_{0}}^{1}(\Omega)}\right)>F\left(r_{2}\right)>F\left(r_{1}\right)=E(u(0)),
$$

which also contradicts (38) and proves (41).

On the other hand, from the identity of the energy, it holds that

$$
\begin{aligned}
& \frac{1}{2}\|u\|_{H_{\Gamma_{0}}^{1}(\Omega)}^{2} \leq E(u(0))+\frac{1}{p+1}\|u\|_{p+1}^{p+1}-\frac{1}{k+1}\|u\|_{k+1, \Gamma_{1}}^{k+1} \\
& \leq E(u(0))+\frac{1}{p+1}\|u\|_{p+1}^{p+1}+\frac{1}{k+1}\|u\|_{k+1, \Gamma_{1}}^{k+1},
\end{aligned}
$$

which implies, from (37), (41) and by the definition of $F$, that

$$
\begin{aligned}
& \frac{1}{p+1}\|u\|_{p+1}^{p+1}+\frac{1}{k+1}\|u\|_{k+1, \Gamma_{1}}^{k+1} \geq \frac{1}{2}\|u\|_{H_{\Gamma_{0}}^{1}(\Omega)}^{2}-E(u(0)) \\
& \geq \frac{1}{2} r_{1}^{2}-F\left(r_{1}\right)=\frac{c_{*}^{p+1}}{p+1} r_{1}^{p+1}+\frac{B_{*}^{k+1}}{k+1} r_{1}^{k+1}
\end{aligned}
$$

Theorem 3.4. Suppose that the assumption (6) holds, $u(0)=u_{0} \in H_{\Gamma_{0}}^{1}(\Omega)$ and $u$ is a local solution of the system (1), $\left\|u_{0}\right\|_{H_{\Gamma_{0}}^{1}(\Omega)}>r_{0}$ and $E(0)<E_{1}$. Then the solution of problem (1) blows up.

Proof. We set

$$
H(t)=E_{2}-E(t)
$$

where $E_{2}$ is a constant and $E(0)<E_{2}<E_{1}<d$. By the definition of $H(t)$ and (9)

$$
H^{\prime}(t)=-E^{\prime}(t) \geq 0,
$$

which implies that $H(t)$ is non-decreasing, and, consequently,

$$
H(t) \geq H(0)=E_{2}-E(0)>0 .
$$

Considering Lemma 3.3, we have that $\|u(t)\|_{H_{\Gamma_{0}}^{1}(\Omega)} \geq r_{1}$, for some $r_{1}>r_{0}$. From this inequality, the definition of the energy and taking (45) into account, we deduce

$$
\begin{aligned}
& H(t)=E_{2}-\left[\frac{1}{2}\|u\|_{H_{\Gamma_{0}}^{1}(\Omega)}^{2}-\frac{1}{p+1}\|u\|_{p+1}^{p+1}+\frac{1}{k+1}\|u\|_{k+1, \Gamma_{1}}^{k+1}\right] \\
& \leq E_{1}-\frac{1}{2}\|u\|_{H_{\Gamma_{0}}^{1}(\Omega)}^{2}+\frac{1}{p+1}\|u\|_{p+1}^{p+1}-\frac{1}{k+1}\|u\|_{k+1, \Gamma_{1}}^{k+1} \\
& \leq E_{1}-\frac{1}{2} r_{1}^{2}+\frac{1}{p+1}\|u\|_{p+1}^{p+1}-\frac{1}{k+1}\|u\|_{k+1, \Gamma_{1}}^{k+1},
\end{aligned}
$$


which implies, having in mind that $E_{1}=F\left(r_{0}\right)=\frac{1}{2} r_{0}^{2}-\frac{c_{*}^{p+1}}{p+1} r_{0}^{p+1}-\frac{B_{*}^{k+1}}{k+1} r_{0}^{k+1}$, that

$$
\begin{aligned}
& H(t) \leq \frac{1}{2} r_{0}^{2}-\frac{c_{*}^{p+1}}{p+1} r_{0}^{p+1}-\frac{B_{*}^{k+1}}{k+1} r_{0}^{k+1}-\frac{1}{2} r_{1}^{2}+\frac{1}{p+1}\|u\|_{p+1}^{p+1}-\frac{1}{k+1}\|u\|_{k+1, \Gamma_{1}}^{k+1} \\
& \leq-\frac{c_{*}^{p+1}}{p+1} r_{0}^{p+1}-\frac{B_{*}^{k+1}}{k+1} r_{0}^{k+1}+\frac{1}{p+1}\|u\|_{p+1}^{p+1}-\frac{1}{k+1}\|u\|_{k+1, \Gamma_{1}}^{k+1} \\
& \leq \frac{1}{p+1}\|u\|_{p+1}^{p+1}-\frac{1}{k+1}\|u\|_{k+1, \Gamma_{1}}^{k+1} \\
& \leq \frac{1}{p+1}\|u\|_{p+1}^{p+1} \leq \frac{1}{p+1}\|u\|_{p+1}^{p+1}+\frac{1}{k+1}\|u\|_{k+1, \Gamma_{1}}^{k+1}
\end{aligned}
$$

Then we can prove the theorem similar to the proof of Theorem 3.2.

\section{Asymptotic stability}

In this section, we will state and prove the exponential decay of the solutions to problem (1). In this context, we have the following lemma.

Lemma 4.1. Let $u$ be a solution to problem (11). Assume that assumption (6) holds and $u_{0} \in W$, then we have

$$
\begin{aligned}
& \|u\|_{H_{\Gamma_{0}}^{1}(\Omega)}^{2} \leq \frac{2(p+1)}{p-1} E(t) \leq \frac{2(p+1)}{p-1} E(0), \\
& \|u\|_{k+1, \Gamma_{1}}^{k+1} \leq B_{*}^{k+1}\left(\frac{2(p+1)}{p-1} E(0)\right)^{k-2}\|u\|_{H_{\Gamma_{0}}^{1}(\Omega)}^{2}, \\
& \|u\|_{p+1}^{p+1} \leq c_{*}^{p+1}\left(\frac{2(p+1)}{p-1} E(0)\right)^{p-2}\|u\|_{H_{\Gamma_{0}}^{1}(\Omega)}^{2} .
\end{aligned}
$$

Proof. By Lemma 2.5, we have $u \in W$ and $I(u)>0$. We know from (9) and the definition of $E(t)$ that

$$
\begin{aligned}
& E(0) \geq E(u)=\frac{1}{2}\|u\|_{H_{\Gamma_{0}}^{1}(\Omega)}^{2}-\frac{1}{p+1}\|u\|_{p+1}^{p+1}+\frac{1}{k+1}\|u\|_{k+1, \Gamma_{1}}^{k+1} \\
& \geq \frac{1}{2}\|u\|_{H_{\Gamma_{0}}^{1}(\Omega)}^{2}-\frac{1}{p+1}\|u\|_{p+1}^{p+1}+\frac{1}{p+1}\|u\|_{k+1, \Gamma_{1}}^{k+1}+\left(\frac{1}{k+1}-\frac{1}{p+1}\right)\|u\|_{k+1, \Gamma_{1}}^{k+1} \\
& \geq\left(\frac{1}{2}-\frac{1}{p+1}\right)\|u\|_{H_{\Gamma_{0}}^{1}(\Omega)}^{2}+\frac{1}{p+1} I(u)+\left(\frac{1}{k+1}-\frac{1}{p+1}\right)\|u\|_{k+1, \Gamma_{1}}^{k+1} \\
& \geq\left(\frac{1}{2}-\frac{1}{p+1}\right)\|u\|_{H_{\Gamma_{0}}^{1}(\Omega)}^{2}+\frac{p-k}{(k+1)(p+1)}\|u\|_{k+1, \Gamma_{1}}^{k+1} .
\end{aligned}
$$

Thus we obtain (49). By the embedding $H_{\Gamma_{0}}^{1}(\Omega) \hookrightarrow L^{k+1}\left(\Gamma_{1}\right)$ and (49), we have

$$
\|u\|_{k+1, \Gamma_{1}}^{k+1} \leq B_{*}^{k+1}\|u\|_{H_{\Gamma_{0}}^{1}(\Omega)}^{k+1} \leq B_{*}^{k+1}\left(\frac{2(p+1)}{p-1} E(0)\right)^{k-2}\|u\|_{H_{\Gamma_{0}}^{1}(\Omega)}^{2} .
$$


Then, (50) holds. By the embedding $H_{\Gamma_{0}}^{1}(\Omega) \hookrightarrow L^{p+1}(\Omega)$ and (49), we have

$$
\|u\|_{p+1}^{p+1} \leq c_{*}^{p+1}\|u\|_{H_{\Gamma_{0}}^{1}(\Omega)}^{p+1} \leq c_{*}^{p+1}\left(\frac{2(p+1)}{p-1} E(0)\right)^{p-2}\|u\|_{H_{\Gamma_{0}}^{1}(\Omega)}^{2},
$$

Then, we conclude (51). Hence, we complete the proof.

Now, we state an important lemma by Martinez [54].

Lemma 4.2. Let $E: R^{+} \rightarrow R^{+}$be a nonincreasing function. Assume that there exists $\sigma>0$ for which $\int_{S}^{+\infty} E(t) d t \leq \sigma E(S)$ for any $S \geq 0$, then there exist two positive constants $C$ and $\xi$ independent of $t$ such that:

$$
0<E(t) \leq C e^{-\xi t} .
$$

Theorem 4.3. Assume that assumption (6) holds and $u_{0} \in W$. Moreover, assume that $E(0)<d$ and $B_{*}^{k+1}\left(\frac{2(p+1)}{p-1} E(0)\right)^{k-2} \frac{(p+1)(k-1)}{(p-1)(k+1)}+c_{*}^{p+1}\left(\frac{2(p+1)}{p-1} E(0)\right)^{p-2}=$ $\alpha<1$, then there exist two positive constants $\hat{C}$ and $\xi$ independent of $t$ such that:

$$
0<E(t) \leq \hat{C} e^{-\xi t}
$$

Proof. Multiplying the first equation in problem (1D) by $u$, then integrating it over $\Omega \times(S, T)$, and performing several integration by parts, we get:

$$
\begin{aligned}
& \int_{S}^{T} \int_{\Omega}\left[u u_{t}+\nabla u \nabla u_{t}+|u|^{q-2} u u_{t}\right] d x d t+\int_{S}^{T}\|u\|_{H_{\Gamma_{0}}^{1}(\Omega)}^{2} d t \\
& +\int_{S}^{T}\|u\|_{k+1, \Gamma_{1}}^{k+1} d t+k \int_{S}^{T} \int_{\Gamma_{1}}|u|^{k-1} u u_{t} d x d t=\int_{S}^{T}\|u\|_{p+1}^{p+1} d t .
\end{aligned}
$$

From the definition of $E(t)$ and equation (52), we obtain

$$
\begin{aligned}
& 2 \int_{S}^{T} E(t) d t=\int_{S}^{T}\left[\|u\|_{H_{\Gamma_{0}}^{1}(\Omega)}^{2}+\frac{2}{k+1}\|u\|_{k+1, \Gamma_{1}}^{k+1}+\frac{2}{p+1}\|u\|_{p+1}^{p+1}\right] d t \\
& =-\int_{S}^{T} \int_{\Omega}\left[u u_{t}+\nabla u \nabla u_{t}\right] d x d t-\int_{S}^{T} \int_{\Omega}|u|^{q-2} u u_{t} d x d t \\
& -k \int_{S}^{T} \int_{\Gamma_{1}}|u|^{k-1} u u_{t} d x d t-\frac{k-1}{k+1} \int_{S}^{T}\|u\|_{k+1, \Gamma_{1}}^{k+1} d t+\frac{p-1}{p+1} \int_{S}^{T}\|u\|_{p+1}^{p+1} d t .
\end{aligned}
$$

Now, we estimate every term on the right-hand side of (53).

Employing Hölder's inequality, Young's inequality, (49) and (91), the first and second terms on the right-hand side of (53) can be estimated as follows, for $\delta_{1}>0$,

$$
\begin{aligned}
& -\int_{S}^{T} \int_{\Omega}\left[u u_{t}+\nabla u \nabla u_{t}\right] d x d t \leq \delta_{1} \int_{S}^{T}\|u\|_{H_{\Gamma_{0}}^{1}}^{2}(\Omega) \\
& \leq \delta_{1} \frac{2(p+1)}{p-1} \int_{S}^{T} E(t) d t-C\left(\delta_{1}\right) \int_{S}^{T} E^{\prime}(t) d t
\end{aligned}
$$


By Hölder's inequality, Young's inequality, (49) and (9), the third terms on the right-hand side of (53) can be estimated as follows, for $\delta_{2}>0$,

$$
\begin{aligned}
& -\int_{S}^{T} \int_{\Omega}|u|^{q-2} u u_{t} d x d t=-\int_{S}^{T} \int_{\Omega}\left(|u|^{\frac{q-2}{2}} u_{t}\right)|u|^{\frac{q}{2}} d x d t \\
& \leq \delta_{2} \int_{S}^{T}\|u\|_{q}^{q} d t+C\left(\delta_{2}\right) \int_{S}^{T} \int_{\Omega}|u|^{q-2} u_{t}^{2} d x d t \\
& \leq \delta_{2} c_{*}^{q} \frac{2(p+1)}{p-1}\left(\frac{2(p+1)}{p-1} E(0)\right)^{q-2} \int_{S}^{T} E(t) d t-C\left(\delta_{2}\right) \int_{S}^{T} E^{\prime}(t) d t
\end{aligned}
$$

where we used the embedding $H_{\Gamma_{0}}^{1}(\Omega) \hookrightarrow L^{q}(\Omega)$ and (49).

Similar to the process of the proof of (55) and by (50), we have

$$
\begin{aligned}
& -\int_{S}^{T} \int_{\Gamma_{1}}|u|^{k-1} u u_{t} d x d t \leq \int_{S}^{T} \int_{\Gamma_{1}}|u|^{\frac{k+1}{2}}\left(|u|^{\frac{k-1}{2}} u_{t}\right) d x d t \\
& \leq \delta_{3} \int_{S}^{T}\|u\|_{k+1, \Gamma_{1}}^{k+1} d t+C\left(\delta_{3}\right) \int_{S}^{T} \int_{\Gamma_{1}}|u|^{k-1} u_{t}^{2} d x d t \\
& \leq \delta_{3} B_{*}^{k+1}\left(\frac{2(p+1)}{p-1} E(0)\right)^{k-2} \frac{2(p+1)}{p-1} \int_{S}^{T} E(t) d t-C\left(\delta_{3}\right) \int_{S}^{T} E^{\prime}(t) d t(56)
\end{aligned}
$$

As for the fifth term on the right-hand side of (53), by (50) and (9), we arrive at

$$
\begin{aligned}
& -\frac{k-1}{k+1} \int_{S}^{T}\|u\|_{k+1, \Gamma_{1}}^{k+1} d t \\
& \leq 2 B_{*}^{k+1}\left(\frac{2(p+1)}{p-1} E(0)\right)^{k-2} \frac{(p+1)(k-1)}{(p-1)(k+1)} \int_{S}^{T} E(t) d t .
\end{aligned}
$$

For the sixth term on the right-hand side of (53), by (50) and (9), we get

$$
\frac{p-1}{p+1} \int_{S}^{T}\|u\|_{p+1}^{p+1} d t \leq 2 c_{*}^{p+1}\left(\frac{2(p+1)}{p-1} E(0)\right)^{p-2} \int_{S}^{T} E(t) d t .
$$

Then, combining these estimates (54)-(58), (53) becomes

$$
\begin{aligned}
& 2 \int_{S}^{T} E(t) d t \\
& \leq\left[\delta_{1} \frac{2(p+1)}{p-1}+\delta_{2} c_{*}^{q} \frac{2(p+1)}{p-1}\left(\frac{2(p+1)}{p-1} E(0)\right)^{q-2}\right. \\
& +\delta_{3} B_{*}^{k+1}\left(\frac{2(p+1)}{p-1} E(0)\right)^{k-2} \frac{2(p+1)}{p-1} \\
& +2 B_{*}^{k+1}\left(\frac{2(p+1)}{p-1} E(0)\right)^{k-2} \frac{(p+1)(k-1)}{(p-1)(k+1)}
\end{aligned}
$$




$$
\begin{aligned}
& \left.+2 c_{*}^{p+1}\left(\frac{2(p+1)}{p-1} E(0)\right)^{p-2}\right] \int_{S}^{T} E(t) d t \\
& -\left(C\left(\delta_{1}\right)+C\left(\delta_{2}\right)+C\left(\delta_{3}\right)\right) \int_{S}^{T} E^{\prime}(t) d t .
\end{aligned}
$$

Note $B_{*}^{k+1}\left(\frac{2(p+1)}{p-1} E(0)\right)^{k-2} \frac{(p+1)(k-1)}{(p-1)(k+1)}+c_{*}^{p+1}\left(\frac{2(p+1)}{p-1} E(0)\right)^{p-2}=\alpha<1$, and choose $\delta_{1}>0, \delta_{2}>0, \delta_{3}>0$ sufficiently small such that

$$
\begin{aligned}
2 & -\delta_{1} \frac{2(p+1)}{p-1}-\delta_{2} c_{*}^{q} \frac{2(p+1)}{p-1}\left(\frac{2(p+1)}{p-1} E(0)\right)^{q-2} \\
& -\delta_{3} B_{*}^{k+1}\left(\frac{2(p+1)}{p-1} E(0)\right)^{k-2} \frac{2(p+1)}{p-1}-2 \alpha>0 .
\end{aligned}
$$

Hence, by (9), there exists a positive constant $\sigma>0$ such that

$$
\int_{S}^{T} E(t) d t \leq \sigma E(S), \text { for any } S \geq 0 .
$$

By letting $T$ go to $+\infty$ on the left hand in the aforementioned inequality, one can easily deduce that Lemma 4.2 is satisfied. Hence the conclusion of Theorem 4.3 is established.

By Lemma 4.1, we have the following result

Corollary 4.4. Under the assumption of Theorem 4.3, there exist two positive constants $C$ and $\xi$ independent of $t$ such that:

$$
\|u\|_{H_{\Gamma_{0}}^{1}(\Omega)} \leq C e^{-\xi t}
$$

Remark 4.5. If $g(u)$ is boundary source term and $f(u)$ is absorptive term, we can also get the similar results.

\section{Conclusions}

This paper consider the initial boundary value problem of the generalized Boussinesq equation with nonlinear interior source and boundary absorptive terms. Under appropriate assumptions imposed on the source and boundary absorption terms, we establish global existence of solutions by using the potential well method combined with a standard continuous argument and we give sufficient conditions for the blow-up of solutions with positive and negative initial energy respectively in a finite time. It is different with the results in 4, 5]. We also give a general decay of the energy by an integral inequality in [54].

\section{Acknowledgements}

This work is supported by the National Natural Science Foundation of China (No.11801145).

\section{Competing Interests}

The authors declare that they have no competing interests. 


\section{REFERENCES}

1. Sveshnikov, A. G., Alshin, A. B., Korpusov, M. O., \& Pletner, Y. D. (2007). Linear and nonlinear equations of Sobolev type. Fizmatlit, Moscow.

2. Korpusov, M. O., \& Sveshnikov, A. G. (2008). Sufficient close-to-necessary conditions for the blowup of solutions to a strongly nonlinear generalized Boussinesq equation. Computational Mathematics and Mathematical Physics, 48(9), 1591-1599.

3. Al'shin, A. B., Korpusov, M. O., \& Sveshnikov, A. G. (2011). Blow-up in nonlinear Sobolev type equations (Vol. 15). Walter de Gruyter.

4. Korpusov, M. O., \& Sveshnikov, A. G. (2008). Sufficient conditions for the blowup of a solution to the Boussinesq equation subject to a nonlinear Neumann boundary condition. Computational Mathematics and Mathematical Physics, 48(11), 2077-2080.

5. Makarov, P. A. (2012). Blow-Up of the solution of the initial boundary-value problem for the generalized Boussinesq equation with nonlinear boundary condition. Mathematical Notes, 92(3-4), 519-531.

6. Levine, H. A. (1973). Some nonexistence and instability theorems for solutions of formally parabolic equations of the form $P u_{t}=-A u+F(u)$. Archive for Rational Mechanics and Analysis, 51(5), 371-386.

7. Kalantarov, V. K., \& Ladyzhenskaya, O. A. (1978). The occurrence of collapse for quasilinear equations of parabolic and hyperbolic types. Journal of Soviet Mathematics, 10(1), 53-70.

8. Karch, G. (1997). Asymptotic behaviour of solutions to some pseudoparabolic equations. Mathematical Methods in the Applied Sciences, 20(3), 271-289.

9. Benjamin, T. B., Bona, J. L., \& Mahony, J. J. (1972). Model equations for long waves in nonlinear dispersive systems. Phil. Trans. R. Soc. Lond. A, 272(1220), 47-78.

10. Sun, C., \& Yang, M. (2008). Dynamics of the nonclassical diffusion equations. Asymptotic Analysis, 59(1-2), 51-81.

11. Payne, L. E., \& Sattinger, D. H. (1975). Saddle points and instability of nonlinear hyperbolic equations. Israel Journal of Mathematics, 22(3-4), 273-303.

12. Sattinger, D. H. (1968). On global solution of nonlinear hyperbolic equations. Archive for Rational Mechanics and Analysis, 30(2), 148-172.

13. Yacheng, L., \& Junsheng, Z. (2006). On potential wells and applications to semilinear hyperbolic equations and parabolic equations. Nonlinear Analysis: Theory, Methods 8 Applications, 64(12), 2665-2687.

14. Xu, R., \& Su, J. (2013). Global existence and finite time blow-up for a class of semilinear pseudo-parabolic equations. Journal of Functional Analysis, 264(12), 2732-2763.

15. Korpusov, M. O., \& Sveshnikov, A. G. (2006). Blow-up of solutions of nonlinear Sobolev type equations with cubic sources. Differential Equations, 42(3), 431-443.

16. Korpusov, M. O. (2004). Blow-up of solutions of a class of strongly non-linear equations of Sobolev type. Izvestiya: Mathematics, 68(4), 783-832.

17. Deng, K., \& Levine, H. A. (2000). The role of critical exponents in blow-up theorems: the sequel. Journal of Mathematical Analysis and Applications, 243(1), 85-126.

18. Messaoudi, S. A. (2002). A note on blow up of solutions of a quasilinear heat equation with vanishing initial energy. Journal of mathematical analysis and applications, 273(1), 243-247.

19. Levine, H. A., Park, S. R., \& Serrin, J. (1998). Global existence and nonexistence theorems for quasilinear evolution equations of formally parabolic type. Journal of Differential Equations, 142(1), 212-229.

20. Di, H., \& Shang, Y. (2015). Global existence and nonexistence of solutions for the nonlinear pseudo-parabolic equation with a memory term. Mathematical Methods in the Applied Sciences, 38(17), 3923-3936.

21. Peng, X., Shang, Y., \& Zheng, X. (2016). Blow-up phenomena for some nonlinear pseudoparabolic equations. Applied Mathematics Letters, 56, 17-22. 
22. Chen, H., \& Tian, S. (2015). Initial boundary value problem for a class of semilinear pseudo-parabolic equations with logarithmic nonlinearity. Journal of Differential Equations, 258(12), 4424-4442.

23. Luo, P. (2015). Blow-up phenomena for a pseudo-parabolic equation. Mathematical Methods in the Applied Sciences, 38(12), 2636-2641.

24. Conti, M., \& Marchini, E. M. (2016). A remark on nonclassical diffusion equations with memory. Applied Mathematics \&5 Optimization, 73(1), 1-21.

25. Korpusov, M. O., \& Sveshnikov, A. G. (2005). Blow-up of solutions of a class of strongly non-linear dissipative wave equations of Sobolev type with sources. Izvestiya: Mathematics, 69(4), 733-770.

26. Aristov, A. I. (2014). Modelling unsteady processes in semiconductors using a non-linear Sobolev equation. Izvestiya: Mathematics, 78(3), 427-442.

27. Zhang, H., Lu, J., \& Hu, Q. (2014). Exponential growth of solution of a strongly nonlinear generalized Boussinesq equation. Computers 83 Mathematics with Applications, 68(12), $1787-1793$

28. Lu, J., Hu, Q., \& Zhang, H. (2014). Blowup of Solution for a Class of Doubly Nonlinear Parabolic Systems. Journal of Function Spaces, 2014, Article ID:924596.

29. Eden, A., Michaux, B., \& Rakotoson, J. M. (1991). Doubly nonlinear parabolic-type equations as dynamical systems. Journal of Dynamics and Differential Equations, 3(1), 87-131.

30. ElOuardi, H., \& ElHachimi, A. (2006). Attractors for a class of doubly nonlinear parabolic systems. Electronic Journal of Qualitative Theory of Differential Equations, 2006(1), 1-15.

31. Levine, H. A., \& Sacks, P. E. (1984). Some existence and nonexistence theorems for solutions of degenerate parabolic equations. Journal of Differential Equations, 52(2), 135-161.

32. Aristov, A. I. (2012). On the initial boundary-value problem for a nonlinear Sobolev-type equation with variable coefficient. Mathematical Notes, 91(5-6), 603-612.

33. Korpusov, M. O. (2013). Solution blow-up for a class of parabolic equations with double nonlinearity. Sbornik: Mathematics, 204(3), 323-346.

34. Truong, L. X., \& Van Y, N. (2016). Exponential growth with $L^{p}$-norm of solutions for nonlinear heat equations with viscoelastic term. Applied Mathematics and Computation, $273,656-663$.

35. Truong, L. X., \& Van Y, N. (2016). On a class of nonlinear heat equations with viscoelastic term. Computers 83 Mathematics with Applications, 72(1), 216-232.

36. Levine, H. A., Smith, R. A., \& Payne, L. E. (1987). A potential well theory for the heat equation with a nonlinear boundary condition. Mathematical Methods in the Applied Sciences, 9(1), 127-136.

37. Vitillaro, E. (2005). Global existence for the heat equation with nonlinear dynamical boundary conditions. Proceedings of the Royal Society of Edinburgh Section A: Mathematics, 135(1), 175-207.

38. Fiscella, A., \& Vitillaro, E. (2011). Local Hadamard well-posedness and blow-up for reaction-diffusion equations with non-linear dynamical boundary conditions. Discrete and Continuous Dynamical Systems, 33 (11), 5015-5047.

39. Amann, H. (1988). Parabolic evolution equations and nonlinear boundary conditions. Journal of Differential Equations, 72(2), 201-269.

40. Escher, J. (1989). Global existence and nonexistence for semilinear parabolic systems with nonlinear boundary conditions. Mathematische Annalen, 284(2), 285-305.

41. Escher, J. (1993). Quasilinear parabolic systems with dynamical boundary conditions. Communications in Partial Differential Equations, 18(7-8), 1309-1364.

42. Escher, J. (1995). On the qualitative behaviour of some semilinear parabolic problems. Differential and Integral Equations, 8(2), 247-267.

43. Carvalho, A. N., Oliva, S. M., Pereira, A. L., \& Rodriguez-Bernal, A. (1997). Attractors for parabolic problems with nonlinear boundary conditions. Journal of Mathematical Analysis and Applications, 207(2), 409-461. 
44. Cavalcanti, M. M., Cavalcanti, V. N. D., \& Lasiecka, I. (2007). Well-posedness and optimal decay rates for the wave equation with nonlinear boundary dampingsource interaction. Journal of Differential Equations, 236(2), 407-459.

45. Lasiecka, I., \& Tataru, D. (1993). Uniform boundary stabilization of semilinear wave equations with nonlinear boundary damping. Differential Integral Equations, 6(3), 507-533.

46. Vitillaro, E. (2002). A potential well theory for the wave equation with nonlinear source and boundary damping terms. Glasgow Mathematical Journal, 44(3), 375-395.

47. Vitillaro, E. (2002). Global existence for the wave equation with nonlinear boundary damping and source terms. Journal of Differential Equations, 186(1), 259-298.

48. Bociu, L., \& Lasiecka, I. (2010). Local Hadamard well-posedness for nonlinear wave equations with supercritical sources and damping. Journal of Differential Equations, 249(3), 654-683.

49. Bociu, L., Rammaha, M., \& Toundykov, D. (2011). On a wave equation with supercritical interior and boundary sources and damping terms. Mathematische Nachrichten, 284(16), 2032-2064.

50. Wu, S. T. (2012). General decay and blow-up of solutions for a viscoelastic equation with nonlinear boundary damping-source interactions. Zeitschrift fr angewandte Mathematik und Physik, 63(1), 65-106.

51. Wu, S. T. (2014). Blow-up of positive initial energy solutions for a system of nonlinear wave equations with supercritical sources. Journal of Dynamical and Control Systems, 20(2), 207-227.

52. Said-Houari, B., \& Nascimento, F. A. F. (2013). Global existence and nonexistence for the viscoelastic wave equation with nonlinear boundary damping-source interaction. Communications on Pure \& Applied Analysis, 12(1), 375-403.

53. Graber, P. J., \& Said-Houari, B. (2012). Existence and asymptotic behavior of the wave equation with dynamic boundary conditions. Applied Mathematics \& Optimization, 66(1), 81-122.

54. Martinez, P. (1999). A new method to obtain decay rate estimates for dissipative systems. ESAIM: Control, Optimisation and Calculus of Variations, 4, 419-444.

55. Lions, J. L. (1969). Quelques methodes de resolution des problemes aur limites non lineaires. Dunod Gauthier-villars, Paris.

56. Vitillaro, E. (1999). Global nonexistence theorems for a class of evolution equations with dissipation. Archive for Rational Mechanics and Analysis, 149(2), 155-182.

\section{Jian Dang}

Department of Mathematics, Henan University of Technology, Zhengzhou 450001, China. e-mail: dangjian2006@163.com

\section{Qingying $\mathrm{Hu}$}

Department of Mathematics, Henan University of Technology, Zhengzhou 450001, China. e-mail: slxhqy@163.com

\section{Hongwei Zhang}

Department of Mathematics, Henan University of Technology, Zhengzhou 450001, China. e-mail: whz661@163.com 